\section{Sylvie Duvernoy}

Via Benozzo Gozzoli, 26 50124 Florence Italy syld@kimwilliamsbooks.com

Keywords: architecture and mathematics, Nexus conference
Conference Report

\section{Nexus VII: Relationships Between Architecture and Mathematics}

Point Loma Nazarene University, San Diego, USA 23-25 June 2008

Abstract. Sylvie Duvernoy reports on the seventh international, interdisciplinary Nexus conference for architecture and mathematics.

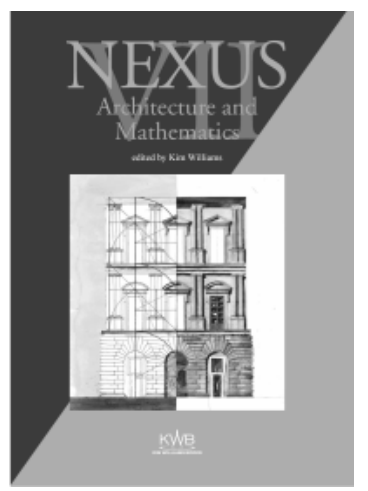

The seventh biennial conference on Architecture and Mathematics "Nexus VII" took place in June 2008, hosted by the Point Loma Nazarene University, in San Diego California, the first Nexus conference held in the United States. Professor Maria Zack, the Chair of the Department of Mathematical, Information and Computer Sciences was the efficient director of the on-site organizing committee, as well as a member of the scientific committee.

The scientific committee organized the conference following the same pattern that was successfully applied during the previous conferences: arranging papers in thematic sessions that covered fields such as representation, history, non-Western architecture, twentieth-century design analysis, survey, and astronomy. Some themes were similar to those that were addressed in the previous conferences, but some others were new, like survey and astronomy.

Representation is a recurrent field of research in the Nexus conferences and in the pages of the Nexus Network Journal. Here lies indeed the very link - the very nexus - between architecture and mathematics. Representation is made possible thanks to the act of drawing, but "drawing" is a generic term that covers a vast array of different graphic operations, and therefore its connections with science and art are multi-faceted. In architecture, "drawing" intended as "drafting" refers to the mathematical rules of descriptive and projective geometries; while "drawing" intended as "designing" refers to mathematical patterns that span from the classic planar geometric grids up to the modern $3 \mathrm{D}$ generative software programs. The history of representation shows that this very special nexus between architecture and mathematics has always existed, evolving in form but not in essence. Two papers addressed these issues, each focusing on drawing as architecture's conceptual medium, but dealing with two different historical periods. Kristina Luce analysed Raphael's Renaissance drawing of the Pantheon, and Ning Gu presented the modern 3D virtual environment design tool.

The topics discussed in the history session were also closely related to representation since they ranged from the history of perspective (João Pedro Xavier), to the scientific analysis of Villalpando's drawings of the ideal architecture of Solomon's Temple (Tessa Morrison), and the proportions in Alberti's Sant'Andrea (Michael Ytterberg).

Nexus Network Journal 11 (2009) 135-138

NEXUS NETWORK JOURNAL - VOL. 11, NO. 1, 2009135

1590-5896/09/010135-05 DOI 10.1007/s00004-008-0108-8

(C) 2009 Kim Williams Books, Turin 
While approaching twentieth-century design analysis from the standpoint of relationship between architecture and mathematics, the researchers often face the same difficulty which consists in the absence of written evidences that would suggest or confirm their hypothesis. Nothing like the many Renaissance architectural treatises that emphasized the necessity for pure proportions and/or symbolic numbers, is available in the twentieth-century literature about architecture. Except for the Modulor by le Corbusier, in contemporary architectural books, the social, economic, innovative technological aspects of architecture often eclipse the formal design recipes that remain in the intimate, private and unspoken part of the designer's mind. This difficulty is particularly obvious to the scholars of Louis Kahn. There are many written texts that report the architectural concerns of the great master (both original manuscripts and transcriptions of conferences), but none of these ever refers to mathematics. Nonetheless, the shapes of many of Louis Kahn's most beautiful buildings show proportions that can hardly be considered to be random. Louis Kahn's works (discussed by Steven Fleming and Mark Reynolds) illustrate a certain kind of contemporary approach to geometrical patterns and classical proportions which is characterized by the search for a mere loose visual image. The final aesthetic result does not rely on a strict adherence to a rule, but admits a certain degree of liberty and deliberate approximation.

During the same session, Michael Ostwald presented a paper (prepared with Josephine Vaughan and Chris Tucker) about a new possible analysis tool, based on the measurement of the fractal complexity of twentieth-century architecture. The houses by Frank Lloyd Wright and le Corbusier were the samples to which they applied their tool and discussed the results. It is not the first time that fractal geometry triggers the curiosity of the scholars involved in the study of the relationships between architecture and mathematics; however until now very few studies had been really convincing. On the contrary, the rigor and the clarity of the methodology proposed there shows how this kind of approach may turn into a new, valuable evaluation tool.

For the first time in a Nexus Conference, the schedule included a session on astronomy. Similarly to all other sessions, this session was international. Two papers presented the relationship between astronomy and single monuments: two observatories whose function defined their shape. Maria Zack described Robert Hooke's commemorative monument to the great fire of London which conceals a telescope on top; and Jang Hoon Kim and Sang Hun Park tried to unveil the mysteries of an oriental medieval stone masonry monument. In a third paper Niels Bandholm approached the topic on a larger territorial scale, investigating the peculiar distribution of the medieval churches in the Danish island of Bornholm and pointing out some parallels with the projection of the celestial sphere on the astrolabe. These researches (together with the ones that hopefully will follow) brilliantly complete the array of classical studies to which Nexus is dedicated. So far architecture had been related to arithmetic, geometry and music but never to the last of the four classical liberal arts: astronomy.

The proceedings of the Conference gather all of the papers that were presented, except for three. Two of these appear in the present issue of the Nexus Network Journal, and the third is forthcoming. The book is simply entitled: Nexus VII: Architecture and Mathematics" and is the latest addition to the series of the previous six Nexus books.

Kim Williams, the editor, says in her introduction, "to best represent these papers I have arranged them in groups, allowing papers that treat similar subjects to be easily contrasted and compared". However, the groups in the proceedings do not exactly match the 
conference sessions. In fact some papers often deal with more than one of the many relationships between architecture and mathematics. This is true, for instance, for the presentations that discuss survey data. The survey methodology and the data interpretation make use of two different kinds of relationships between mathematics and architecture: one is technological, and the other is intellectual. The research may be more oriented towards the evaluation of the survey methodology that produced the data, its appropriateness and its limits, especially when digital technology and laser scanners are involved. On the other hand, the discussion may focus on the data themselves, and their interpretation from the point of view of geometrical design. This is why in the book of the proceedings, the papers presented in the "Survey" session of the conference are scattered in various chapters: the laser-scan surveys of Irish architecture conducted by Maurice Murphy, Sara Pavia and Eugene Mc Govern are found in the "Digital Technology" chapter, while the study of the Medieval Irish churches by Avril Behan and Rachel Moss is under "Western Design Analyses".

Also, the papers gathered in the "Historical Analyses" chapter all deal with representation, and could therefore be compared from more than one standpoint.

Both the conference works and the book of the proceedings show once more how rich and multi-faceted the nexus between architecture and mathematics is. The development and evolution of the analyses tools bring progress and evolution in knowledge and culture, triggering new discussions and researches.

The next Nexus conference will take place in Porto (Portugal), 13-15 June 2010.

\section{About the reviewer}

Sylvie Duvernoy is the Book Review Editor of the Nexus Network Journal. She has participated at five consecutive Nexus conferences (Ferrara 2000, Obidos 2002, Mexico City 2004, Genoa 2006 and San Diego 2008). 